\title{
Psychiatric Disorder in the Community: Disease or Distress?
}

\author{
Report of a Meeting of the Social and Community Psychiatry Section
}

\author{
E. S. PAYKel. Professor of Psychiatry, St. Gcorge's Hospital Medical School, London SW17 \\ and J. P. LefF. Assistant Dircctor. MRC Social Psychiatry Unit, Friern Hospital, London $N 11$
}

\begin{abstract}
The Section of Social and Community Psychiatry held a well attended scientific meeting on 24 Februrary 1984 in London. The aim was to examine recent evidence on the frequency of psychiatric disorder in the community and the debate as to its nature. Many studies have suggested relatively high rates, raising the question as to whether what is being identified is akin to the disorders treated by psychiatrists or represents something milder and qualitatively nearer to normal distress. Speakers were George Brown, Paul Bebbington, Norman Kreitman, Eugene Paykel. Glenys Parry and David Goldberg.
\end{abstract}

Professor G. W. Brown suggested that to invoke distress rather than discase as an explanation for the frequency of depression in the community or its association with loss or failure represented an unhelpful pigeon-holing of conditions as cither biological or psychological, rather than possibly multifactorial. Current research indicated that a large number of so-called distress conditions were seen by psychiatrists; that such conditions showed many of the core symptoms of clinical depression: that in the general population at any one time half of the cases of depression had lasted continuously for at least one year; that the majority of all forms of depression, whether or not seen by psychiatrists, were provoked by life crises; and that forms of depression could not convincingly be distinguished by the presence or absence of such crises, although there was some evidence for a very modest association. Professor Brown presented data from his current Islington study of a random sample of working class women indicating that nearly one-fifth of women experienced definite depression within a year. The severity of depression was not greater among the small minority of women who saw a psychiatrist although they showed other characteristics such as suicide gestures, accompanying alcoholism and drug abuse. The data suggested that patients and nonpatients were not easily distinguished by severity of depression but by other features which made them more conspicuous to the general practitioner and at the same time less easy to deal with.

Dr P. Bebbington pointed out that, as a branch of medicine, psychiatry had many approaches to its subject matter but the one which marked it out was the setting up and testing of disease theories. The categories used in this process were initially syndromal and were useful in so far as they could be shown to establish a consistent aetiology, pathology, treatment or management. Such categories were always to some extent tentative. This raised the question as to when depressed mood fitted into such a catcgory. Disease theorics of depression dated from the nineteenth century. Pathological depression, it was suggested, could be recognized because it was likely to have unusual accessory symptoms and to be relatively unaffected by the social environment. Bequeathed such ideas, there was an obligation to test them. In their Camberwell survey he and colleagues had found that cases in the gencral population were more likely to be related to preceding adversity and less likely to show 'endogenous' symptoms than cases attending psychiatric facilities. Moreover, lack of adversity was associated with the presence of accessory symptoms. However, the latter findings did run counter to the consensus of the literature which showed only a minor decrease in adversity in depression with endogenous symptoms. It still remained possible that disorders in the community were more likely to have been provoked by adversity than were disorders in psychiatric clinics. Dr Bebbington also presented emerging preliminary data from a study of endogenous symptoms and adversity in out-paticnts. currently under way.

Dr N. KREtTman criticized introduction of the criterion of understandability into discussions of caseness. Case criteria based on presence of specific psychological characteristics were highly reliable. Use of caseness and understandability on a mutually exclusive basis implied that nobody could have an understandable illness. The act of 'understanding' cut across standard scientific enquiry in that it was based on intuitive perceptions which could not be made explicit. Jaspers' distinction between types of understanding was meant to concern kinds of explanation rather than differences between individuals. On the other hand much closer attention than hitherto should be paid to non-cases in population studies. A substantial number of these were 'demoralized' individuals who had distressing psychological experiences or symptoms which were insufficient to warrant an illness diagnosis. This group should be delineated and studied. Three approaches were possible. Firstly, if demoralization represented a minor form of psychiatric illness then one would expect in longitudinal studies over time that demoralized persons would be especially likely to move in and out of the illness category. Preliminary tests on a survey of Edinburgh women which he presented suggested that the demoralized were in fact at much higher risk of being clinically ill on a later occasion or conversely to have recently been ill. A second prediction was that sub-groups in the population showing high rates of cases would also show high rates of demoralization. In this respect the evidence was weak; in Professor Brown's data there was no connection between the epidemiological profiles of his cases and borderline cases. The Edinburgh data, although different, were only weakly supportive of the continuum view. Finally one could ask whether the causal factors believed to generate illness also generated demoralization. Drs Ingham and Miller in Edinburgh had found 
in a general practice survey that the life events, social support and demographic variables which conduced to frank clinical illness also, in lesser doses, made for states of non-specific anxiety and depression. There was a hint of a threshold or 'catastrophe' effect so that a small increase in stressors might lead to a major change in severity of psychopathology. So far, therefore, studies tended to support a unitary concept but conclusions would be premature and a rich research field was available.

Professor E. S. PAYkel pointed out that available evidence indicated that the majority of psychiatric cases within the community saw their general practitioners within the same year, although the disorder might be unrecognized. Studies of general practice consulters could therefore throw light on the qualities of community disorders. He presented data from a study of general practice depression carried out from St. George's with colleagues. Three samples were studied: depressives stanted on a new course of an antidepressant by their GPs; depressives identified by the doctor and given other treatment: and Research Diagnostic Criteria (RDC) major depressives missed by the GP but identified by screening and subsequent interview. They were studied with multiple rating instruments including the PSE, RDC, Hamilton Depression Scale. The majority were cases on the Index of Definition but predominantly at or just above threshold level and 29 per cent of the other treatment sample were non-cases. Among the antidepressant treated sample, about half were RDC major depsessives and a quarter, minor or intermittent depressives; but among those given other treatment only 20 per cent were major depressives and 30 per cent were minor depressives, while the remainder had anxiety or other diagnoses. The antidepressant treated sample showed considerably less severe depression and less evidence of endogenous symptoms than depressed psychiatric out-patients. The missed major depressives tended to show less overt depressive symptomatology than identified major depressives, but differences were relatively small. Overall the findings indicated that, although it mainly satisfied diagnostic criteria, depression in general practice differed considerably in severity and quality from that treated by psychiatrists.

Ms G. PARRY presented data from the Sheffield Mothers Project (MRC/ESRC Social and Applied Psychology Unit) which studied the effects of paid employment, life event stress and social support on the mental health of working class women with young children. In addition to the PSE as a case identification procedure, a number of continuously distributed indices of psychological distress were used, including the Beck Depression Inventory. Zung Self-Rated Depression Scale, and measures of somatic anxiety, positive and negative affect and self-esteem. It was found that there was a discontinuity in the linear relationship between the continuously distributed measures and Index of Definition level, between ID4 and ID5. The categorical model was then applied to the data by comparing respondents in ID6 to 8 (definite cases) with those at ID1 to ID4 (non-cases). using discriminant analysis. When items were pre-selected to relate to the psychiatric diagnosis of depression, approximately one-third of ID5 (threshold) respondents were classified as 'cases' using a Bayesian weighted procedure. When using general well-being items, this proportion dropped to 12 per cent and, when using self-attitude items, almost all the threshold cases were within the normal range.

The discussion was opened by Professor D. P. Goldberg. There followed a vigorous discussion involving all speakers and with much audience participation. A particular sense of occasion was imparted by the presence of some of the principal protagonists in a scientific debate which has attracted much attention and it will remain a memorable day for those who attended.

\section{A New Instrument for Assessment in Rehabilitation Psychiatry}

REHAB - the Rehabilitation Evaluation Hall and Baker is a recently published method for assessing chronic psychiatric patients. It is intended for use in psychiatric hospitals, day hospitals, hostels and secure institutions. Its authors, John Hall and Roger Baker, are very experienced researchers and practitioners in this ficld and feel that there exists a need in psychiatric rehabilitation for an assessment capable of being applied in a variety of situations. They have therefore constructed REHAB as a multipurpose instrument which can be used: (i) to measure change in patients' behaviour, e.g. for drug trial; (ii) to select in-patients with potential for living in the comunity; (iii) to select disturbed/severely handicapped patients; (iv) to select groups of patients generally: (v) to help plan treatment programmes for individual patients; (vi) to help plan treatment/ intervention/reorganization for a ward, group of wards or whole institution.

REHAB is a package of material containing all that is necessary for assessing up to 50 patients. The entire package consists of a manual for the person administering the assessment, book lets to help raters to understand the assessment, the assessment forms themselves, materials for scoring and recording information and sheets designed for presenting the REHAB findings on an individual patient or group of patients. Different items may be replenished as needed.

More detailed information about REHAB, as well as price list and order form, may be obtained from the publishers, Vine Publishing Lid, 2A Eden Place, Aberdeen AB2 4YF, Scotland. A specimen set containing single copies of various parts of the package, retailing at $£ 5.89$ (inclusive of VAT, postage and packing) may also be obtained from Vine Publishing Ltd. 